\title{
Editorial: Computational Social Science as the ultimate Web Intelligence
}

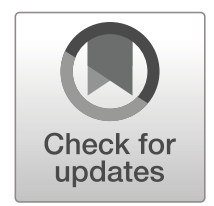

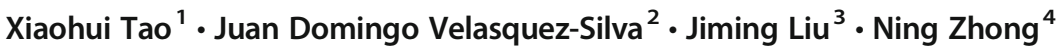 \\ Published online: 4 March 2020 \\ (C) Springer Science+Business Media, LLC, part of Springer Nature 2020
}

Computational Social Science (CSS) is the use of Web Intelligence and the tools and technology capable of monitoring, analyzing, diagnosing, and resolving day-to-day problems of society. CSS is the development of intelligent systems and solutions to address the critical problems of the society such as poverty and hunger, slavery and torture, disease and suffering, and create tools that enable an illiterate person to be as productive as a $\mathrm{PhD}$. The understanding was proposed and advocated by Dabbala Rajagopal ("Raj”) Reddy, who received the ACM Turing Award in 1994 for "pioneering the design and construction of large scaled artificial intelligence systems, demonstrating the practical importance and potential commercial impact of artificial intelligence technology" [1]. In a keynote speech delivered at the IEEE/WIC/ACM International Conference on Web Intelligence in Leipzig, Germany on August 24, 2017, Reddy pointed out that "Computer Science and Artificial Intelligence must embrace CSS as the next frontier in Web intelligence." [2] His vision on Artificial Intelligence and Web Intelligence for creating a truly humane society is both thought-provoking and instrumental to bringing about new revolutions in the two related fields.

This article belongs to the Topical Collection: Computational Social Science as the Ultimate Web Intelligence Guest Editors: Xiaohui Tao, Juan D. Velasquez, Jiming Liu, and Ning Zhong

Xiaohui Tao

xiaohui.tao@usq.edu.au

Juan Domingo Velasquez-Silva

jvelasqu@dii.uchile.cl

Jiming Liu

jiming@comp.hkbu.edu.hk

Ning Zhong

zhong@maebashi-it.ac.jp

1 School of Sciences, University of Southern Queensland, Toowoomba, Australia

2 University of Chile, Santiago, Chile

3 Hong Kong Baptist University, Kowloon Tong, Hong Kong

4 Maebashi Institute of Technology, Maebashi, Japan 
In an interview with Reddy on March 17, 2018 [3], he once again reinforced the vision on new revolutions in the two fields with a focus on Computational Social Science. He raised a number of interesting questions as an inspiration to the WWW and AI related research communities:

- What is your vision of the future of AI?

- What could be the future impact of Web Intelligence?

- How to incorporate Personal Guardian Angels to future Web Intelligence?

- How could we further advance the field of computational social sciences via the route of Web Intelligence?

This Special Issue is an effort and a step taken forward to explore the answers to these important and inspiring questions. The Special Issue consists of 12 articles (selected from 35 submissions) that discussed theories and methodologies from both disciplines of Artificial Intelligence and Web Intelligence with a focus on Computational Social Science and the related methodologies and technologies. The discussions encompass the theoretical basis and related tools to formally represent, measure, model, and mine meaningful patterns from largescale online datasets related to AI, WWW, and Computational Social Science. They are briefly introduced as follows.

The vision of future AI comes from deep understanding of current AI techniques. A survey article, "From Ideal to Reality: Segmentation, Annotation, and Recommendation, the Vital Trajectory of Intelligent Micro Learning," studied state-of-the-art Web technologies and mobile devices and their development trajectory. The study provided a deep, insightful understanding of data sources and intelligent techniques that are widely used on Web Intelligence and Social Computational Sciences.

Computational Social Science may turn into a continuing learning process when social phenomena is viewed as data and transformed to data representation for analysis. "A Continuous Learning Method for Recognizing Named Entities by Integrating Domain Contextual Relevance Measurement and Web Farming Mode of Web Intelligence" is an endeavour departing from such an understanding. The work is a perfect showcase of how Web Intelligence impacts Computational Social Science - via viewing and analysing social phenomena as data in the connected hyper world.

Web social media is an essential information source for studies in Computational Social Science and Web Intelligence. Aiming at promoting our accessibility to Web social media, "Query-based Unsupervised Learning for Improving Social Media Search" proposed a novel model to learn the implicity relationships in the short text from social media and used them to improve Web social media search. Having a different focus, "A Comprehensive Analysis of Adverb Types for Mining User Sentiments on Amazon Product Reviews" studied Web social media for accessibility to user sentiments and opinions through comprehensive understanding of human natural language. "Topic Based Time-Sensitive Influence Maximization in Online Social Networks," however, is a study on the diffusion problem of Web social media on online social networks. These works advanced our methodologies and technologies in representing, measuring, modelling, and mining meaningful patterns from large-scale online resources .

Predicting future trend of Web events can help improve the quality of Web services, especially when social context is taken into account of the prediction algorithm. A work presented in "Web Event Evolution Trend Prediction Based on Its Computational Social Context" developed a computational model for the social context first and adopted it to evaluate the interaction and influence between social context and Web events for trend prediction of the events. 
In order to incorporate Personal Guardian Angels to future Web Intelligence, deep understanding of individual people and their personalities is essential. Personality analysis has been widely used in social services such as mental healthcare, recommendation systems and so on because its natural ex-plainability for AI applications in Web Intelligence. The article "Grouplevel Personality Detection Based on Text Generated Networks" introduced a novel model to detect group-level personality through learning the influence from text generated networks. The work made a significant original contribution to personality trait prediction on the basis of collaborative identification. The authors in "Learning Part-alignment Feature for Person Reidentification with Spatial-temporal-based Re-ranking Method" proposed a network to learn powerful features from different resources for person re-identification, along with a novel reranking method by exploiting the spatial-temporal information. These works have advanced our understanding of human beings and personalities via Web Intelligence and Computational Science perspective.

Many Artificial Intelligence and Machine Learning techniques have been proven promising in the design of Web services and Web based systems. The article "Extractive Convolutional Adversarial Networks for Network Embedding" proposed a deep learning model, aiming at extracting the latent representations from the topological structure, the attributed information and labels. Another article, "Robust SVM With Adaptive Graph Learning," took the dynamic graph learning and the self-paced learning into account and introduced a novel SVM method. "Spectral Clustering via Half-quadratic Optimization" focused on existing spectral clustering issues and presented a new clustering algorithm along with the technique of half-quadratic optimization. In another interesting work, "End-to-End Latent-Variable Task-Oriented Dialogue System with Exact Log-likelihood Optimization," the authors proposed an end-to-end dialogue model based on a hierarchical encoder-decoder and relying on a discrete latent variable to learn underlying dialogue intentions. These works made significant contributions to methodological advancement for Web Intelligence and Computational Social Science.

With aforementioned inspiring works, the Special Issue has shown itself an theoretical and empirical exploration of important and interesting research topics in Computational Social Science via Web Intelligence. The Special Issue forms a valuable reference to academia, researchers, and industrial practitioners who are interested in the recent advancement in Web Intelligence, online social media, and Computational Social Science, the important and emerging research areas in World Wide Web.

\section{References}

1. Association for Computing Machinery. A. M. Turing Award Laureates - Dabbala Rajagopal ("Raj”) Reddy. URL: https://amturing.acm.org/award_winners/reddy_9634208.cfm, Accessed on 20 Jan 2020

2. Sheth, A., Ngonga, A., Wang, G., Chang, E., Slezak, D., Franczyk, B., Alt, R. and Tao, X. (eds.) Proceedings of 17th IEEE/WIC/ACM International Conference on Web Intelligence, 2017 (WI '17), Leipzig Germany August, 2017. ISBN: 9781450349512, Association for Computing Machinery, New York

3. Zhong, N., Liu, J., Shi, Y., Yao, Y.Y.: An interview with Professor Raj Reddy on Web Intelligence (WI) and Computational Social Science (CSS). Web Intelligence. 16(3), 143-146 (2018)

Publisher's note Springer Nature remains neutral with regard to jurisdictional claims in published maps and institutional affiliations. 